\title{
A Continuation Semantics of Interrogatives That Accounts for Baker's Ambiguity
}

Chung-chieh Shan

Harvard University

Wh-phrases in English can appear both raised and in-situ. However, only in-situ $w h$-phrases can take semantic scope beyond the immediately enclosing clause. I present a denotational semantics of interrogatives that naturally accounts for these two properties. It neither invokes movement or economy, nor posits lexical ambiguity between raised and in-situ occurrences of the same wh-phrase. My analysis is based on the concept of conTINUATIONS. It uses a novel type system for higher-order continuations to handle wide-scope $w h$-phrases while remaining strictly compositional. This treatment sheds light on the combinatorics of interrogatives as well as other kinds of so-called $\overline{\mathrm{A}}$-movement.

\section{Introduction}

Baker (1968) discusses multiple-wh questions such as those in (1).

a. Who remembers where we bought what?

b. Who do you think remembers what we bought for whom?

Each question in (1) contains three $w h$-phrases and is ambiguous between two readings with different notions of what constitutes an appropriate answer.

(2) Who remembers where we bought what?

a. Alice remembers where we bought the vase.

b. Alice remembers where we bought what.

(3) Who do you think remembers what we bought for whom?

a. I think Alice remembers what we bought for Bob.

b. I think Alice remembers what we bought for whom.

Intuitively, both cases of ambiguity are because the final wh-phrase-what in (1a) and whom in (1b)—can take either wide scope (2a, 3a) or narrow scope $(2 \mathrm{~b}, 3 \mathrm{~b}) .^{1}$

In this paper, I focus on two properties of interrogatives.

- Wh-phrases appear both raised and in-situ. For example, in (1b), who and what appear raised while whom appears in-situ.

- Raised wh-phrases must take semantic scope exactly over the clause they are raised to overtly. For example, in (1b), who must take wide scope, and what must take narrow scope. Only whom has ambiguous scope; accordingly, the question has only 2 readings, not 4 or $8 .^{2}$ 
I present a strictly compositional semantics of interrogatives in English that accounts for these properties. Specifically, in my analysis,

- there is no covert movement or $w h$-raising between surface syntax and denotational semantics (contra Epstein's (1992) economy account), yet a single denotation suffices for both raised and in-situ appearances of each $w h$-phrase. Moreover,

- as a natural consequence of the denotation of wh-phrases and the rules of the grammar, only in-situ wh-phrases can take scope ambiguously.

I describe my system below as one where, roughly speaking, interrogative clauses denote functions from answers to propositions (an old idea). However, such denotations are not crucial for my purposes-the essential ideas in my analysis carry over easily to a system where interrogative clauses denote say sets of propositions instead. Hence this paper bears not so much on what interrogatives denote, but how.

My analysis builds upon Barker's (2000a, 2000b) use of continUaTions to characterize quantification in natural language. In Section 2, I introduce continuation semantics as a two-step generalization of Montague's (1974) treatment of quantification. The system I present generalizes Barker's semantics in several aspects, which I point out below as we encounter. In Section 3, I specify denotations for interrogative elements and account for the properties above. In doing so, I am not concerned with the semantics of verbs such as know that take interrogative complements, but rather with how to derive denotations for interrogative clauses themselves. Finally, in Section 4, I conclude with some speculations on further applications of my treatment, for example to explain superiority effects.

This paper loosely follows the framework of Combinatory Categorial Grammar (Steedman 1987, 1996). However, I expect the central insights to be easy to adapt to other frameworks of compositional semantics.

\section{From PTQ to Continuations in Two Steps}

Continuations are a well-known and widely applied idea in computer science. Many analogies have been drawn to explain the concept; for example, in programming language semantics, it is often said that "the continuation represents an entire (default) future for the computation" (Kelsey, Clinger, Rees et al. 1998).

From the perspective of natural language semantics, a continuation can be thought of as "a semantic value with a hole". To illustrate, consider the sentence

Alice loves Bob.

In Montague grammar, the meaning of (4) is computed compositionally from denotations assigned to Alice, loves, and Bob. Starting with the values

$$
\text { ALICE }: e, \quad \text { LOVE }: e \rightarrow e \rightarrow t, \quad \text { вОв : } e,
$$

where $e$ is the base type of individuals and $t$ is the base type of propositions, we recursively combine semantic values by function application (Figure 1) to obtain 


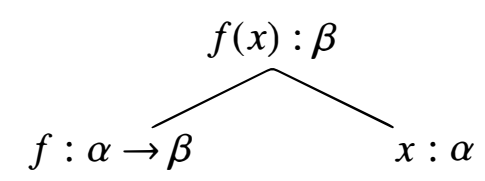

FIGURE 1. Function application ${ }^{3}$

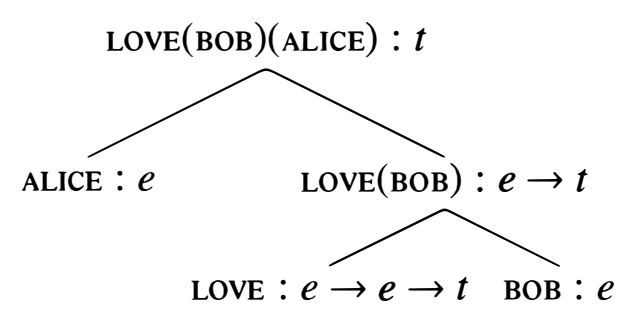

FIGURE 2. Alice loves Bob

the top-level denotation LOVE(вов)(ALICE), of type $t$ (Figure 2). ${ }^{3}$ Borrowing computer science terminology, I say that the EVALUATION CONTEXT of the constituent $B o b$ in the sentence (4) is the "sentence with a hole"

Alice loves _.

Semantically, this evaluation context is essentially a map

$$
c=\lambda x \cdot \operatorname{LOVE}(x)(\text { ALICE })
$$

from individuals to propositions - in particular, the map $c$ sends the individual вов to the proposition LOVE(вов)(aLICE). The map $c$ is called the continUation of Bob in (4). I assign it the type

$$
e \rightarrow t
$$

where $\rightarrow$ is a binary type constructor.

The VALUE TYPE of a continuation is its domain, and the ANSWER TYPE of a continuation is its codomain. For example, the continuation $c$ has value type $e$ and answer type $t .^{4}$ I distinguish between the continuation type $e \rightarrow t$ and the function type $e \rightarrow t$, even though they may be interpreted the same way model-theoretically (standardly, as sets of functions). In fact, I use the same notation to construct and apply functions (namely $\lambda$ and parentheses) as for continuations. The purpose of distinguishing between continuations and functions is to maintain mental hygiene and rule out undesirable semantic combination (see Section 3.2 below).

With the continuation $c$ in (7) in hand, we can apply it то вов to recover the proposition that Alice loves Bob, or apply it to CAROL to generate the proposition that Alice loves Carol. We can play "what-if" with the hole in (6), plugging in different individuals to see what proposition the top-level answer would come out to be. In particular, we can compute

$$
\forall x . c(x)
$$


to generate the proposition that Alice loves every individual. This intuition is why Montague's (1974) "Proper Treatment of Quantification" (PTQ) assigns essentially the denotation

$$
\llbracket \text { everyone }=\lambda c . \forall x . c(x):(e \rightarrow t) \rightarrow t
$$

to the quantificational NP everyone. ${ }^{5}$

Note that the type in (10) is $(e \rightarrow t) \rightarrow t$ rather than the more familiar $(e \rightarrow t) \rightarrow t$. This type documents our intuition that the denotation of everyone is a function that maps each proposition with an $e$-hole (type $e-t$ ) to a proposition with no hole (type $t$ ). In general, a semantic value whose type is of the form $\alpha \rightarrow \gamma$ can be thought of as "an $\gamma$ with a $\alpha$-hole", in other words a continuation that-given a hole-filler of type $\alpha$-promises to produce an answer of type $\gamma$. To redeem this promise is to feed the continuation to a function of type $(\alpha \rightarrow \gamma) \rightarrow \gamma^{\prime}$ in return for a final answer of type $\gamma^{\prime}$. (In the case of everyone in (10), the two answer types $\gamma$ and $\gamma^{\prime}$ are both $t$, and the value type $\alpha$ is $e$.)

As one might expect from this discussion, types of the form $(\alpha \rightarrow \gamma) \rightarrow \gamma^{\prime}$ recur throughout this paper. I write $\alpha\left[\begin{array}{l}\gamma^{\prime} \\ \gamma\end{array}\right]$ as shorthand for such a type. For example, the denotation of everyone given in (10) can be alternatively written

$$
\llbracket \text { everyone } \rrbracket=\lambda c . \forall x . c(x): e\left[\begin{array}{l}
t \\
t
\end{array}\right]
$$

I call $\alpha$ the VALUE TYPE, $\gamma$ the INCOMING ANSWER TYPE, and $\gamma^{\prime}$ the OUTGOING ANSWER TYPE. two steps:

Continuation semantics can be understood as a generalization of PTQ, in

- Lift not just the semantic type of NPs from $e$ to $e\left[\begin{array}{l}t \\ t\end{array}\right]$, but also the semantic type of other phrases from say $\alpha$ to $\alpha\left[{ }_{t}^{t}\right]$.

- Lift each type $\alpha$ to not just the type $\alpha\left[\begin{array}{l}t \\ t\end{array}\right]$, but any type of the form $\alpha\left[\begin{array}{l}\gamma^{\prime} \\ \gamma\end{array}\right]$, where $\gamma$ and $\gamma^{\prime}$ are types.

I detail these steps below.

\subsection{First Generalization: From e $\left[\begin{array}{l}t \\ t\end{array}\right]$ to $\alpha\left[\begin{array}{c}t \\ t\end{array}\right]$}

In PTQ, the semantic type of NPs is not $e$ but $e\left[{ }_{t}^{t}\right]$. For example, the NPs Alice and $B o b$ denote not the individuals ALICE $: e$ and вов $: e$, but rather the lifted values

$$
\begin{aligned}
\llbracket \text { Alice } \rrbracket & =\lambda c . c(\text { ALICE }): e\left[\begin{array}{l}
t \\
t
\end{array}\right], \\
\llbracket \text { Bob } \rrbracket & =\lambda c . c(\text { вов }): e\left[\begin{array}{l}
t \\
t
\end{array}\right] .
\end{aligned}
$$

In general, any value $x: e$ can be lifted to the value $\lambda c . c(x): e\left[\begin{array}{l}t \\ t\end{array}\right]$. The lifted type $e\left[\begin{array}{l}t \\ t\end{array}\right]$ is borne by all NPs, from proper names like Alice and Bob to quantificational NPs such as everyone and someone.

$$
\begin{aligned}
\llbracket \text { everyone } \rrbracket & =\lambda c . \forall x . c(x): e\left[\begin{array}{l}
t \\
t
\end{array}\right], \\
\llbracket \text { someone } \rrbracket & =\lambda c . \exists x . c(x): e\left[\begin{array}{l}
t \\
t
\end{array}\right] .
\end{aligned}
$$




$$
\begin{gathered}
\lambda c . c(x): \alpha\left[\begin{array}{l}
t \\
t
\end{array}\right] \\
x: \alpha
\end{gathered}
$$

FIGURE 3. Lifting semantic values

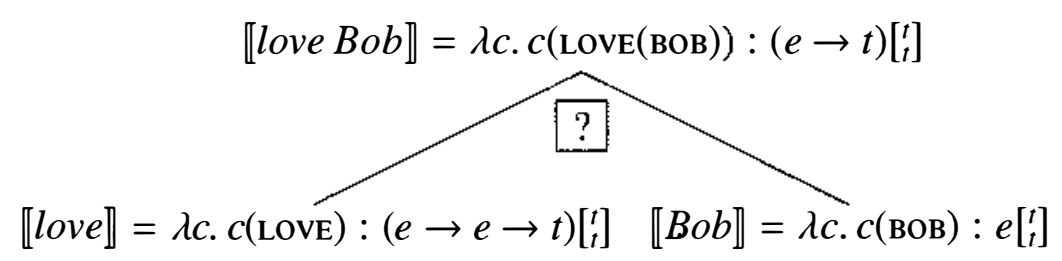

FIGURE 4. The desired output of a lifted semantic rule

Although proper names and quantificational NPs share the same lifted type, the denotations of the latter do not result from lifting any value.

PTQ is appealing in part because it assigns the same lifted type to all NPs, quantificational or not. We can generalize this uniformity beyond NPs. For example, let us lift intransitive verbs from the type $e \rightarrow t$ to $(e \rightarrow t)\left[{ }_{t}^{t}\right]$, and transitive verbs from the type $e \rightarrow e \rightarrow t$ to $(e \rightarrow e \rightarrow t)\left[{ }_{t}^{t}\right]$.

$$
\begin{aligned}
\llbracket \text { smoke } \rrbracket & =\lambda c . c(\text { SMOKE }):(e \rightarrow t)\left[\begin{array}{l}
t \\
t
\end{array}\right], \\
\llbracket \text { love } \rrbracket & =\lambda c . c(\text { LOVE }) \quad:(e \rightarrow e \rightarrow t)\left[\begin{array}{l}
t \\
t
\end{array}\right] .
\end{aligned}
$$

In general, any semantic value $x$, say of type $\alpha$, can be lifted to the value $\lambda c . c(x)$, of type $\alpha\left[\begin{array}{l}t \\ t\end{array}\right]$. This lifting rule is shown in Figure 3.

To maintain the uniformity of types across the grammar, we want every VP to take the same semantic type $(e \rightarrow t)\left[\begin{array}{l}t \\ t\end{array}\right]$. Furthermore, just as the new denotation of smoke in (14a) is its old denotation sMOKE lifted, the new denotation of love Bob should also be its old denotation, LOVE(вов), lifted. What we now need is a semantic rule that will combine a lifted function with a lifted argument to form a lifted result. For example, the rule should combine $\llbracket$ love $\rrbracket=\lambda c . c($ LOVE $)$ with $\llbracket B o b \rrbracket=\lambda c . c($ вов $)$ to form $\lambda c . c(\operatorname{LOVE}($ вов $))$, the denotation we desire for love Bob. This situation is depicted in Figure 4.

Consider now the following calculation.

$$
\begin{aligned}
\lambda c . c(\operatorname{LOVE}(\mathrm{BOB})) & =\lambda c .\left(\lambda c^{\prime} . c^{\prime}(\mathrm{LOVE})\right)(\lambda f . c(f(\mathrm{BOB}))) \\
& =\lambda c . \llbracket \text { love } \rrbracket(\lambda f . c(f(\mathrm{BOB}))) \\
& =\lambda c . \llbracket \text { love } \rrbracket\left(\lambda f .\left(\lambda c^{\prime} . c^{\prime}(\text { вов })\right)(\lambda x . c(f(x)))\right) \\
& =\lambda c . \llbracket \text { love } \rrbracket(\lambda f . \llbracket \text { Bob } \rrbracket(\lambda x . c(f(x)))) .
\end{aligned}
$$

In the first two lines, the atom Love is replaced with a variable $f$, which gets its value from the lifted denotation of love. In the last two lines, the atom вов is similarly replaced with $x$, which gets its value from the lifted denotation of $B o b$. The end result is a way to write down the lifted result of a function application in terms of the lifted function and the lifted argument, without mentioning any unlifted atoms. This 


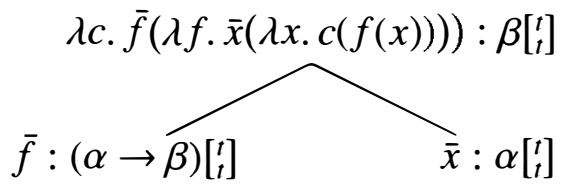

FIGURE 5. Lifted function application (evaluating function then argument)

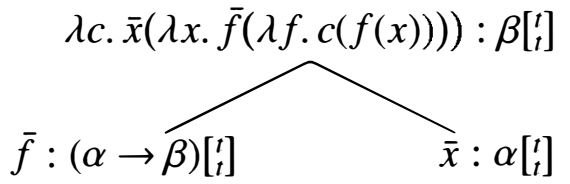

FIGURE 6. Lifted function application (evaluating argument then function)

technique generalizes to a new semantic rule, LIFTED FUNCTION APPLICATION, shown in Figure 5. It satisfies the requirement in Figure 4, as is easily checked.

As it turns out, there is another way to satisfy the requirement. The calculation in (15) above replaces the atom LOVE first and the atom вов second. If instead we replace вов first and Love second, we arrive at a different result.

$$
\begin{aligned}
\lambda c . c(\operatorname{LOVE}(\text { вов })) & =\lambda c \cdot\left(\lambda c^{\prime} \cdot c^{\prime}(\text { вов })\right)(\lambda x \cdot c(\operatorname{LOVE}(x))) \\
& =\lambda c . \llbracket B o b \rrbracket(\lambda x . c(\operatorname{LOVE}(x))) \\
& =\lambda c . \llbracket B o b \rrbracket\left(\lambda x .\left(\lambda c^{\prime} \cdot c^{\prime}(\operatorname{LOVE})\right)(\lambda f . c(f(x)))\right) \\
& =\lambda c . \llbracket B o b \rrbracket(\lambda x . \llbracket \operatorname{love} \rrbracket(\lambda f . c(f(x)))) .
\end{aligned}
$$

This alternative calculation in turn gives rise to a different lifted function application rule, that in Figure 6.

The two rules in Figures 5 and 6 differ in EVALUATION ORDER. Roughly speaking, the evaluation order of a programming language is the order in which "computational side effects" like input and output occur as expressions are evaluated (in other words as code is executed). Continuations are often used in programming language semantics to model evaluation order, for instance in Plotkin's (1975) seminal work. Adopting this terminology, I say that our first rule evaluates the function before the argument, and our second rule evaluates the argument before the function.

\subsection{Quantification}

With the semantic rules and lexical denotations introduced so far, we can derive the sentence Alice loves everyone. Figure 7 shows one analysis, essentially that of Barker's (2000a).

I indicate semantic rules used in derivations with the following notation.

- A unary branch decorated with $\wedge$ invokes the lifting rule (Figure 3 ).

- A binary branch decorated with $>$ invokes the one of the two lifted function application rules that evaluates the left daughter first, in other words either the function-then-argument rule (Figure 5) or the mirror image of the argument-then-function rule (Figure 6).

- A binary branch decorated with < invokes the other of the two lifted function application rules, which evaluates the right daughter first. 


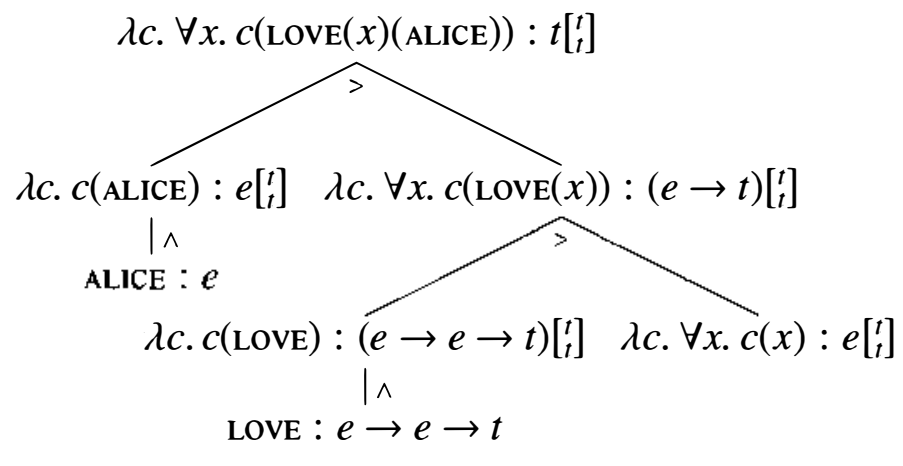

FIGURE 7. Alice loves everyone

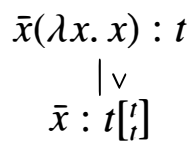

FIGURE 8. Lowering lifted propositions

In this derivation, all evaluation orders give the same result, so both binary branches in Figure 7 could have been decorated with $<$ instead of $>$. Evaluation order does not matter for this sentence because it only contains one quantificational NP. For other sentences, such as someone loves everyone, different orders of evaluation give differently scoped results (Barker 2000a).

\subsection{Lowering}

The top-level denotation derived in Figure 7 has type $t\left[\begin{array}{l}t \\ t\end{array}\right]$, in other words $(t \rightarrow t) \rightarrow t$. This type is not $t$, the type that clauses usually receive in Montague grammar. This discrepancy is expected, since every type $\alpha$, say $\alpha=t$, has been lifted to the type $\alpha\left[\begin{array}{l}t \\ t\end{array}\right]=t\left[\begin{array}{l}t \\ t\end{array}\right]$. We can recover the usual, type- $t$ denotation of the sentence by applying the lifted denotation - as a function - to the identity continuation. That is, compute

$$
(\lambda c . \forall x \cdot c(\operatorname{LOvE}(x)(\operatorname{ALICE})))(\lambda x \cdot x)=\forall x \cdot \operatorname{LOVE}(x)(\operatorname{ALICE}): t
$$

to recover the propositional meaning of Alice loves everyone. Here the identity continuation $\lambda x . x: t \rightarrow t$ corresponds to the trivial evaluation context " ".: a sentence with a sentence hole, and nothing else.

In general, from any value $\bar{x}$ whose type is $t\left[\begin{array}{l}t \\ t\end{array}\right]$ we can recover the propositional value $\bar{x}(\lambda x . x)$, of type $t$. This gives us the LOWERING rule in Figure 8 . Where this rule is used in derivations below, I decorate the unary branch with $\vee$.

\subsection{Second Generalization: From $\alpha\left[\begin{array}{l}t \\ t\end{array}\right]$ to $\alpha\left[\begin{array}{l}\gamma^{\prime} \\ \gamma\end{array}\right]$}

The type $t$ plays a special role in PTQ: It is the only answer type. Because PTQ is after all a treatment of quantification, it is natural there for the answer type to be fixed at $t$ by lexical denotations like those of everyone and someone in (13). However, in the treatment of extraction and interrogation that I present below, not 


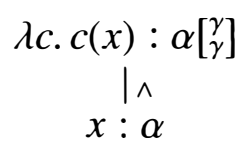

Figure 9. Lifting semantic values, revised

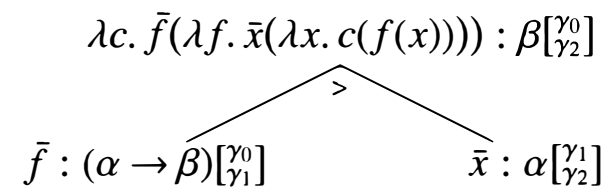

FIGURE 10. Lifted function application (evaluating function then argument), revised

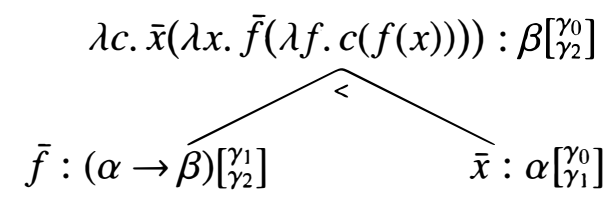

FIGURE 11. Lifted function application (evaluating argument then function), revised

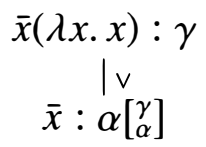

FIGURE 12. Lowering lifted values, revised

all clauses have the same type. Gapped and interrogative clauses do not denote propositions; they have types other than $t$. So our semantic rules must deal with lifted values whose answer types are not $t$.

The semantic rules introduced so far (Figures 3, 5, 6, and 8) do not mention any logical operator. Thus the type $t$ plays no essential role in these rules, and can be replaced with a type variable $\gamma$. Start with the lifting rule (Figure 3): Any semantic value $x$, say of type $\alpha$, can be lifted to the value $\lambda c . c(x)$, of (polymorphic) type $\alpha\left[\begin{array}{l}\gamma \\ \gamma\end{array}\right]$. This revised lifting rule is shown in Figure 9.

The other semantic rules can be similarly revised, by substituting $\gamma$ for $t$ throughout. However, further generalization is possible. We can not only support answer types other than $t$, but also allow multiple answer types to occur in the same derivation. In technical terms, consider the $\lambda$-terms in our semantic rules: How polymorphic can their types be without risking a mismatch? The most general types that can be assigned are shown in Figures 10-12. (Note that the two versions of lifted function application now differ in their types.)

Let me summarize the semantics we have arrived at. Alongside of function application, we have added to Montague grammar four semantic rules: lifting, two versions of lifted function application, and lowering. These four rules, shown in Figures 9-12, suffice below to analyze extraction and interrogation, except $w h$-phrases taking wide scope call for higher-order continuations (Section 3.4). 


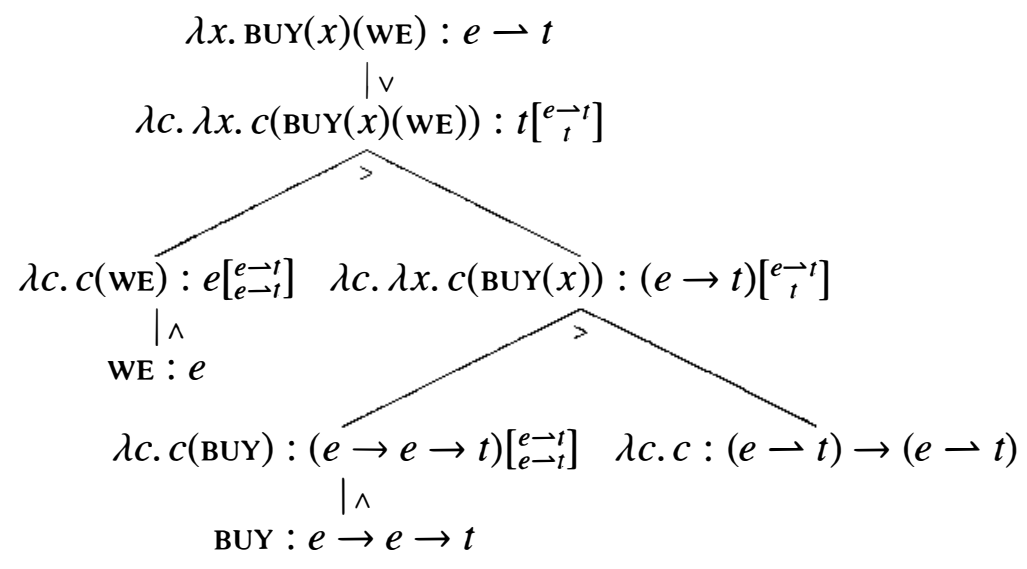

FIGURE 13. We bought

\section{Manipulating Answer Types}

In this section, I present my analysis of interrogatives using continuations. I first analyze extraction and raised $w h$-phrases, then turn to in-situ $w h$-phrases and multiple$w h$ interrogatives.

\subsection{Extraction}

In order to analyze interrogatives with raised $w h$-phrases, I first need a theory of extraction. Fortunately, continuation semantics provides for a natural and compositional analysis of extraction. One possible implementation is to posit a phonologically null NP, notated ".,", whose denotation is ${ }^{6}$

$$
\llbracket-\rrbracket=\lambda c . c:(e \rightarrow \gamma) \rightarrow(e-\gamma)
$$

that is, the identity function over individual-taking continuations. ${ }^{7}$ Figure 13 shows how to derive we bought $\ldots$. The final denotation has type $e \rightarrow t$, a continuation type. This is typical of a clause with an unsaturated gap, and is intuitive considering that continuations are supposed to model evaluation contexts, in other words expressions with holes.

Figure 13 reflects one important difference between the continuation semantics here and Barker's system. Barker takes the answer type to be $t$ everywhere; he does not generalize PTQ to arbitrary answer types (as I do in Section 2.4 above). My present purposes require that we not fix a single answer type, for two reasons. First, not all clauses have the same type. Although basic declarative sentences such as Alice loves Bob have type $t$, I want sentences with gaps and interrogatives to have other semantic types. Second, my semantics not only calls for a mixture of different answer types, but in fact contains denotations that modify what can be thought of as the "current answer type".

Manipulation of answer types is exemplified by the denotation of _ in (18) and the derivation of we bought - in Figure 13. Examining the denotation of , we see that it takes as input an $e$-taking continuation whose answer type is $\gamma$, but 
returns a final answer of type $e \rightarrow \gamma$ instead. Informally speaking, [-』 acts like an $e$ locally, but in addition prepends " $e \rightarrow$ " to the current answer type; hence we

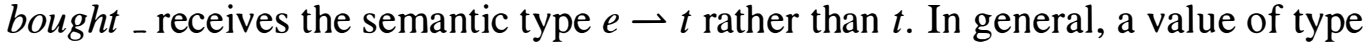

$$
(\alpha \rightarrow \gamma) \rightarrow \gamma^{\prime}
$$

acts like the value type $\alpha$ locally, but in addition transforms the incoming answer

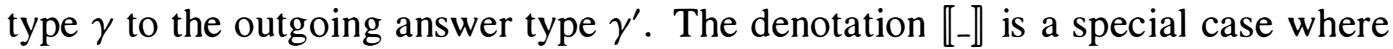
$\alpha=e$ and $\gamma^{\prime}=e \rightarrow \gamma$. Another special case is denotations lifted using the lifting rule, for which $\gamma=\gamma^{\prime}$ and manipulation of answer types degenerates into propagation. ${ }^{8}$

The intuition that values like [-】 "change the current answer type" is reflected my shorthand notation for continuation types, introduced above in Section 2. The type of [- \ can be written alternatively as $e\left[\begin{array}{c}e-\gamma \\ \gamma\end{array}\right]$, so as to emphasize that it acts locally like an $e$, but prepends " $e \rightarrow$ " to the answer type. Note also, in Figures 10 and 11, how lifted function application concatenates two changes to the answer type-first from $\gamma_{2}$ to $\gamma_{1}$, and then from $\gamma_{1}$ to $\gamma_{0}$-into a change from $\gamma_{2}$ to $\gamma_{0}$.

\subsection{Raised Wh-phrases}

As alluded to in Section 1, my interrogative denotations are roughly functions mapping answers to propositions. To make this idea precise, I introduce yet another binary type constructor $\leadsto$, so as to form QUESTION TYPES such as $e \leadsto t$. As before, I distinguish between the question type $e \sim t$, the continuation type $e \rightarrow t$, and the function type $e \rightarrow t$, even though they may have the same models and I overload the same notation to construct and apply all three kinds of abstractions.

I now analyze the sentences

(20) a. Alice remembers what [we bought _].

b. What did we buy _?

I ignore the subject-auxiliary inversion triggered by direct (top-level) interrogatives.

In Section 3.1 is derived a denotation of type $e \rightarrow t$ for the embedded clause we bought _. Let us assume, as is commonly done, that remembrance relates persons (type $e$ ) and questions (type $e \sim t$ for now). Then remember has type $(e \sim t) \rightarrow e \rightarrow t$.

Having assigned meanings to every other word in (20a), I need to specify what what means. Note that we bought - is of type $e \rightarrow t$, but remember requires the distinct type $e \sim t$ for its input. Therefore, the denotation of what should convert we bought _ from $e \rightarrow t$ to $e \leadsto t$. I make the simplest assumption to that effect - that what has the semantic type $(e \rightarrow t) \rightarrow(e \sim t)$. The semantic content of what should be essentially the identity function, but express the requirement that the input to $e \leadsto t$ be somehow inanimate. I am not concerned with the nature of this requirement here, so I simply notate it as a bracketed formula $[\neg \operatorname{ANIMATE}(x)]$, as in

$$
\begin{aligned}
\llbracket w h a t \rrbracket & =\lambda c . \lambda x .[\neg \operatorname{ANimate}(x)] c(x):(e \rightarrow t) \rightarrow(e \sim t), \\
\llbracket w h o \rrbracket & =\lambda c . \lambda x .[\operatorname{animate}(x)] c(x):(e \rightarrow t) \rightarrow(e \sim t) .
\end{aligned}
$$




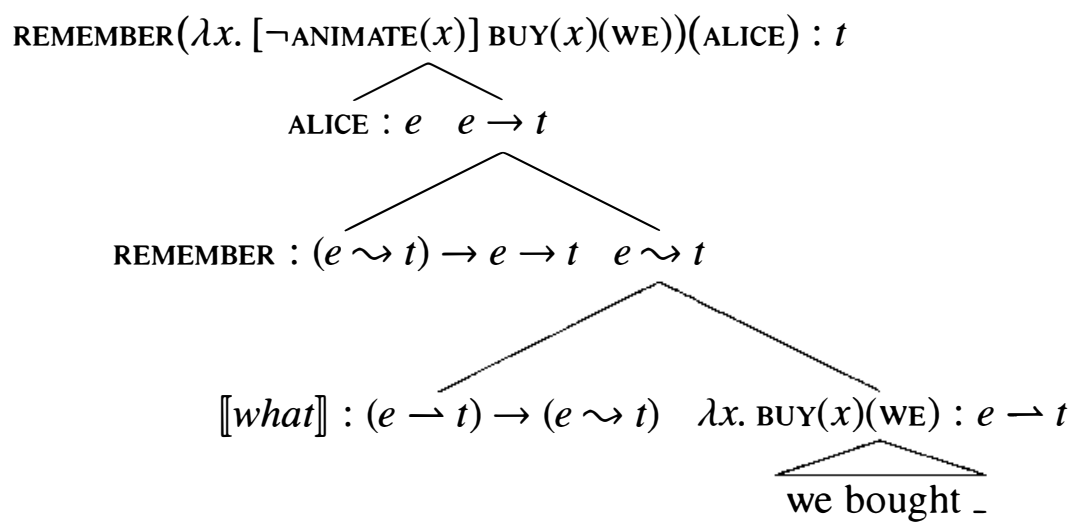

(Figure 13)

FIGURE 14. Alice remembers what [we bought

The formula $[\neg \operatorname{ANIMATE}(x)] c(x)$ can be thought of as "if $\neg \operatorname{ANIMATE}(x)$ then $c(x)$, otherwise undefined".

The definitions in (21) makes no concrete use of the base type $t$. Indeed, we can generalize them to

$$
\begin{aligned}
\llbracket w h a t \rrbracket & =\lambda c \cdot \lambda x .[\neg \operatorname{ANimate}(x)] c(x):(e-\gamma) \rightarrow(e \sim \gamma), \\
\llbracket w h o \rrbracket & =\lambda c . \lambda x .[\operatorname{animate}(x)] c(x):(e-\gamma) \rightarrow(e \sim \gamma) .
\end{aligned}
$$

I use this generalization to analyze multiple-wh clauses in Section 3.3 below. Regardless, we can derive (20a). One derivation is shown in Figure 14. Once the meaning of we bought - is derived, the rest of the derivation consists entirely of (unlifted) function application.

Why distinguish between function types $(\rightarrow)$, continuation types $(\rightarrow)$, and question types $(\sim)$ ? The distinction prevents the grammar from overgenerating sentences like *I remember Alice bought or *I remember what what Alice bought. The types enforce a one-to-one correspondence between gaps and wh-phrasesmore precisely, interrogatives gaps and raised $w h$-phrases.

\subsection{In-situ Wh-phrases}

The analyses above of extraction and raised $w h$-phrases are both natural in the spirit of continuation semantics. The primary payoff from these analyses is that little more needs to be said to treat interrogatives with in-situ $w h$-phrases and to account for the two properties I listed in Section 1.

The first property is that wh-phrases appear both raised and in-situ. For instance, the clauses in (23) contain what raised and whom in-situ.

(23) a. What did we buy _ for whom?

b. what we bought - for whom 


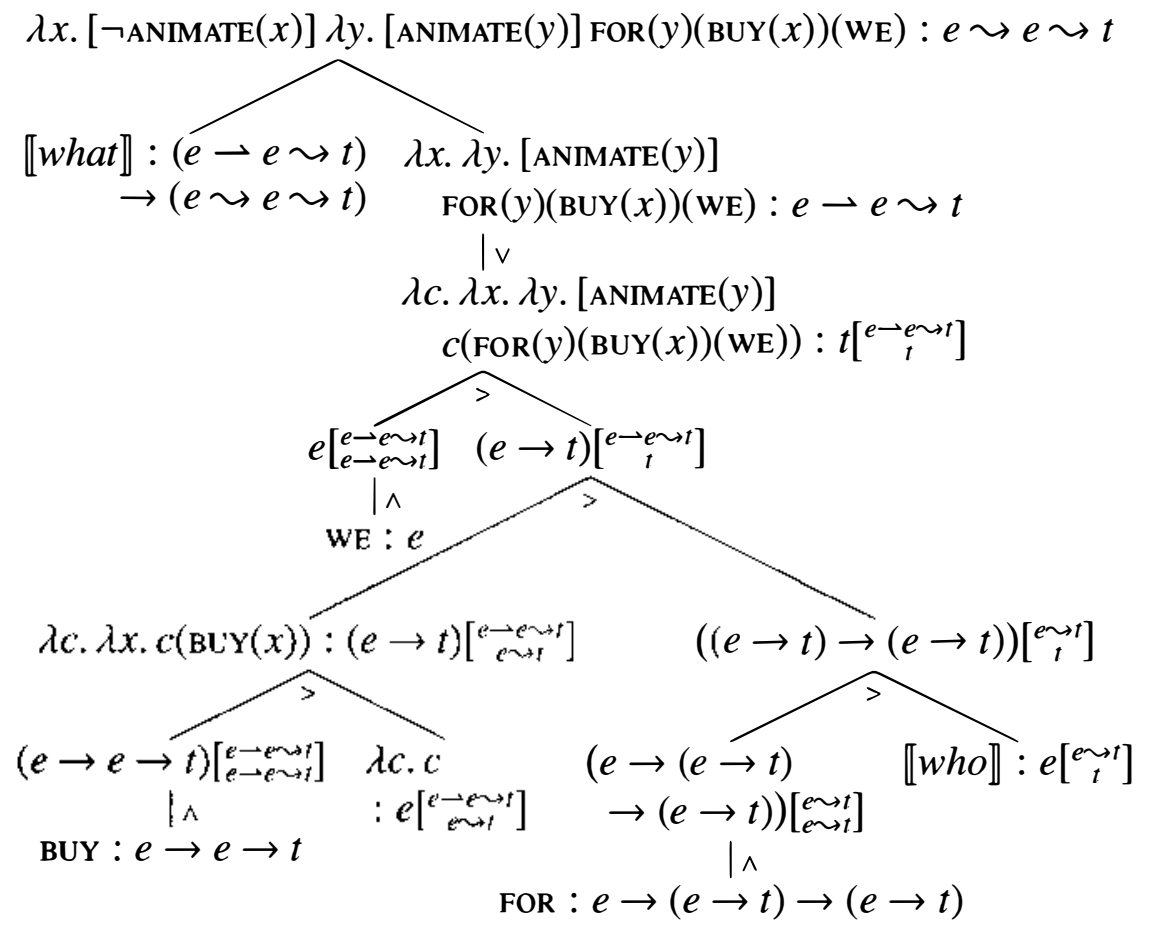

FIGURE 15. What we bought _for whom, with the narrow-scope reading for whom

Ignoring the subject-auxiliary inversion in (23a), these two clauses are identical. To derive them, all we need is an uncontroversial meaning for for:

$$
\llbracket f o r \rrbracket=\mathrm{FOR}: e \rightarrow(e \rightarrow t) \rightarrow(e \rightarrow t)
$$

Given that it was with raised usage in mind that we assigned to whom its meaning in (22b), it may come as a surprise that the same meaning works equally well for in-situ usage. But it does all work out: The derivation, which culminates in the top-level type $e \leadsto e \leadsto t$, is shown in Figure 15. (If we assume furthermore that remember can take semantic type $(e \sim e \sim t) \rightarrow e \rightarrow t$, then it is straightforward to produce the narrow-scope reading (3b) of the example (1b) from Section 1.)

To see how this derivation works, it is useful to examine (22), where what and who were assigned the (polymorphic) type $(e \rightarrow \gamma) \rightarrow(e \sim \gamma)$. In Section 3.2, this type was justified because what needed to convert $(\rightarrow)$ an $e$-taking continuation $(\rightarrow)$ to an $e$-wondering question $(\sim)$. However, the same type can also be written as $e\left[\begin{array}{c}e \leadsto \gamma \\ \gamma\end{array}\right]$. A value of this type takes as input an $e$-taking continuation whose answer is $\gamma$, but returns a final answer of type $e \sim \gamma$ instead. Informally speaking, interrogative NPs act like $e$ s locally, but in addition prepends " $e \sim$ " to the current answer type.

Hence, as one might expect, the double-wh constructions in (23) receive the semantic type $e \leadsto e \leadsto t$. The first " $e \leadsto$ " in the type is contributed by the raised $w h$-phrase what, or rather, contributed as " $e \rightarrow$ " by the extraction gap and subsequently converted to " $e \sim$ " by what. The second " $e \sim$ " in the type is contributed directly by the in-situ wh-phrase whom. Figure 16 illustrates this process. 


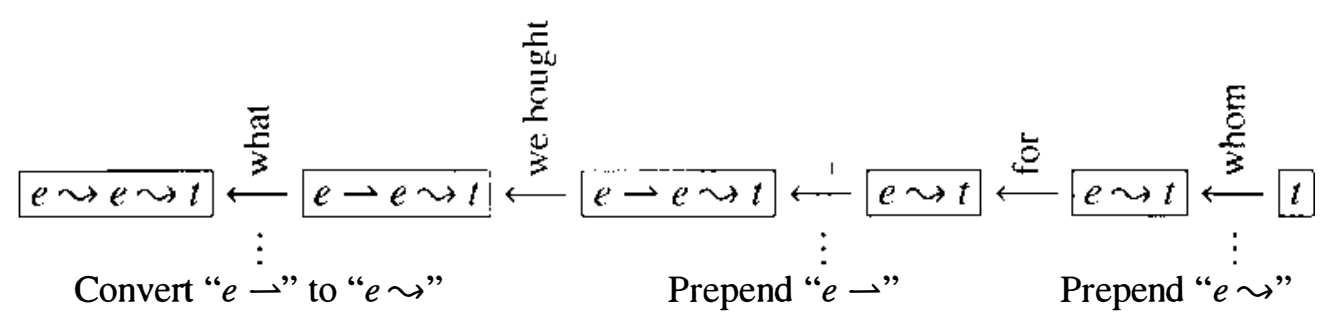

FIGURE 16. Building the semantic type $e \leadsto e \leadsto t$ for what we bought - for whom, with the narrow-scope reading for whom. Whereas the in-situ elements - and whom manipulate the answer type, the raised element what manipulates the value type.

It may be initially perplexing that the types in Figure 16 are manipulated right-to-left. This pattern is explained if we postulate that evaluation in natural language tends to proceed left-to-right. That is, when a function occurs linearly before its argument, the function-then-argument version of lifted function application is preferred, and vice versa. Left-to-right evaluation results in right-to-left answer type manipulation, because constituents to the left decide what the answer type looks like at outer levels. For example, 【-』wants the answer type to be a continuation at the outermost level it gets to affect. When building the answer type bottom-up from $t$ to $e \leadsto e \sim t$, the outermost decisions are executed last, not first.

Critical to the ability of the type $(e \rightarrow \gamma) \rightarrow(e \sim \gamma)$ to serve two roles at once is the lowering rule. In-situ wh-phrases (and gaps) combine with other constituents and manipulate the answer type through lifted function application. By contrast, raised wh-phrases combine with other constituents through ordinary (unlifted) function application, and perform the conversion from " $e \rightarrow$ " to " $e \leadsto$ " not on the answer type but on the value type. Before a raised wh-phrase can act on a gapped clause, then, the clause needs a meaning whose value type-not answer type-is of the form $e-\cdots$. The lowering rule fills this need: It extracts an answer out of a lifted value by feeding it the identity continuation.

\subsection{Higher-Order Continuations}

We have seen above that, in my analysis of interrogatives, a single denotation for each wh-phrase suffices for both raised and in-situ appearances, as long as the wh-phrase takes narrow scope. To fulfill the promises I made in Section 1, I have to turn to wide scope and account for two additional facts about interrogatives: First, I have to show in my system that in-situ wh-phrases can take semantic scope wider than the immediately enclosing clause, as they do in my initial examples (2a) and (3a). Second, I have to show that raised $w h$-phrases cannot take wide scope; in other words, they must take semantic scope exactly where they are overtly located.

I claim that HIGHER-ORDER CONTINUATIONS account for wide scope interrogatives. ${ }^{9}$ Our analyses in previous sections are lifted only to the first order. In PTQ terms, this means that our values are sets of sets; more generally, our types are of the form $\alpha\left[\begin{array}{l}\gamma^{\prime} \\ \gamma\end{array}\right]$. Wide scope calls for lifting to the second order. In PTQ terms, this means that our values need to be sets of sets of sets of sets; more generally, we need to deal with types of the form $\alpha\left[\begin{array}{l}\gamma^{\prime} \\ \gamma\end{array}\right]\left[\begin{array}{l}\delta_{\delta}^{\prime} \\ \delta\end{array}\right]$. Recall that values lifted to the first 
order are manipulated using four additional semantic rules: lifting, lowering, and lifted function application (two versions). I explain below how to introduce further semantic rules into the grammar that manipulate values lifted to higher orders.

As described in Section 2.1, lifted function application is obtained by "lifting" ordinary function application. Since ordinary function application is a binary rule, it can be lifted in two ways (evaluation orders), giving two rules for lifted function application. In general, any $n$-ary semantic rule, schematically

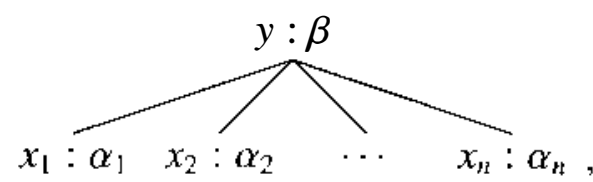

can be lifted in $n$ ! ways, giving rise to $n$ ! lifted rules: For each permutation $\sigma$ of the numbers $1,2, \ldots, n$, we can lift (25) to a new rule

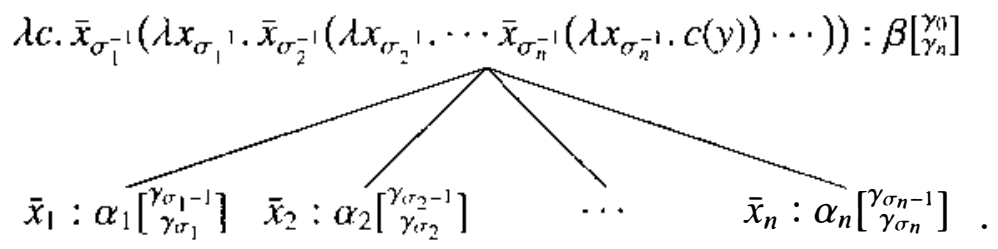

For example, ordinary function application (Figure 1) can be lifted with $\sigma_{1}=1$, $\sigma_{2}=2$ (Figure 10) or with $\sigma_{1}=2, \sigma_{2}=1$ (Figure 11).

Let $G$ be a Montague grammar, each of whose semantic rules are of the form in (25). We can LIFT $G$ to a new grammar $G^{\prime}$, with the following semantic rules:

- the value lifting rule (Figure 9);

- the value lowering rule (Figure 12); and

- every rule in $G$, along with the $n$ ! ways to lift it, where $n$ is the arity.

Let $G_{0}$ be "pure" Montague grammar, where the only semantic rule is function application. Lifting $G_{0}$ gives a new grammar $G_{0}^{\prime}$; call it $G_{1}$. Lifting $G_{1}$ gives another grammar $G_{1}^{\prime}=G_{0}^{\prime \prime}$; call it $G_{2}$. These grammars and their rules are illustrated in Figure 1. The grammar $G_{2}$ contains the semantic rules we need to manipulate values lifted to the second order: lifted lifting, lifted lowering, and twice-lifted function application (four versions). The process may continue indefinitely.

In a once-lifted grammar, many types are of the form $\alpha\left[\begin{array}{c}\gamma^{\prime} \\ \gamma\end{array}\right]$. As explained in Section 3.1, such a type can be understood to mean "acts locally like an $\alpha$ while changing the answer type from $\gamma$ to $\gamma^{\prime \prime}$. In a twice-lifted grammar, many types are of the form $\alpha\left[\begin{array}{c}\gamma^{\prime} \\ \gamma\end{array}\right]\left[\begin{array}{l}\delta^{\prime} \\ \delta\end{array}\right]$. One way to understand such types is to think of a derivation in a twice-lifted grammar as maintaining two answer types - an inner answer type corresponding to the first time the grammar is lifted, and an outer one corresponding to the second time. A type of the form $\alpha\left[\begin{array}{c}\gamma^{\prime} \\ \gamma\end{array}\right]\left[\begin{array}{c}\delta^{\prime} \\ \delta\end{array}\right]$ means "acts locally like an $\alpha$ while changing the inner answer type from $\gamma$ to $\gamma^{\prime}$ and the outer answer type from $\delta$ to $\delta^{\prime \prime}$ ".

To strengthen this understanding, let us examine how answer types are manipulated in the four binary composition rules that result from lifting function application twice. In Figure 3.4, I expand out the rules, in particular the types. In the rules $>^{>}$and $>^{<}$, evaluation proceeds from function to argument at the first contin- 
TABLE 1. Lifting Montague grammar. Starting from function application $\left(G_{0}\right)$, lifting once gives a grammar $\left(G_{1}\right)$ with 2 unary rules and 3 binary rules. Lifting again gives a grammar $\left(G_{2}\right)$ with 4 unary rules and 7 binary rules. In the table, " $f>x$ " means "evaluating function first"; " $x>f$ " means "evaluating argument first".

\begin{tabular}{l} 
Unary rule \\
Value lifting \\
Value lifting, lifted \\
Value lowering \\
Value lowering, lifted \\
\hline Binary rule \\
\hline Function application, lifted $f>x$, lifted $f>x$ \\
Function application, lifted $f>x$ \\
Function application, lifted $f>x$, lifted $x>f$ \\
Function application \\
Function application, lifted $x>f$, lifted $f>x$ \\
Function application, lifted $x>f$ \\
Function application, lifted $x>f$, lifted $x>f$
\end{tabular}

$\left.\left.\lambda d . \overline{\bar{f}}(\lambda \bar{f} \cdot \overline{\bar{x}}(\lambda \bar{x} \cdot d(\lambda c \cdot \bar{f}(\lambda f \cdot \bar{x}(\lambda x \cdot c(f(x))))))): \beta\left[\begin{array}{c}\gamma_{0} \\ \gamma_{2}\end{array}\right]\right]_{\delta_{2}}^{\delta_{0}}\right]$

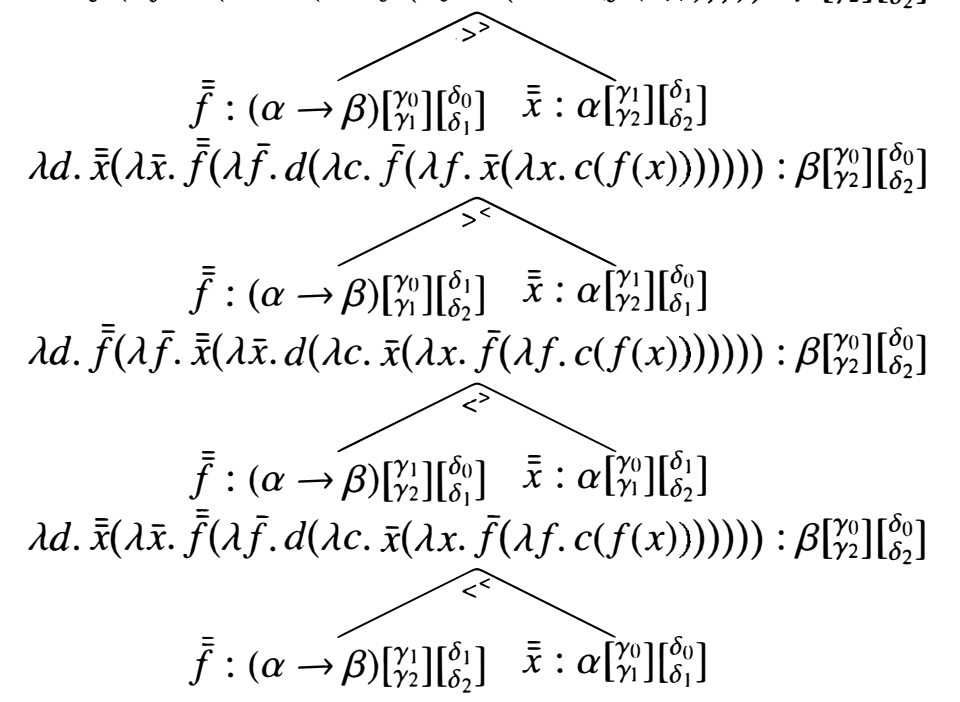

FIGURE 17. Twice-lifted function application (four versions)

uation level. Accordingly, the subscripts on $\gamma$ show that the inner answer type is threaded first through the argument $\overline{\bar{x}}$ and then through the function $\overline{\bar{f}}$. In the rules $<$ and $<$ the reverse happens: Evaluation proceeds from argument to function, and the inner answer type is threaded through first $\overline{\bar{f}}$ and then $\overline{\bar{x}}$.

Similarly for the second continuation level: In the rules $>>$ and $<>$, evaluation proceeds from function to argument, and the subscripts on $\delta$ show that the outer answer type is threaded first through $\overline{\bar{x}}$ and then through $\overline{\bar{f}}$. In the rules $>^{<}$ and $<^{<}$, evaluation proceeds from argument to function, and the outer answer type is threaded through first $\overline{\bar{f}}$ and then $\overline{\bar{x}}$. 


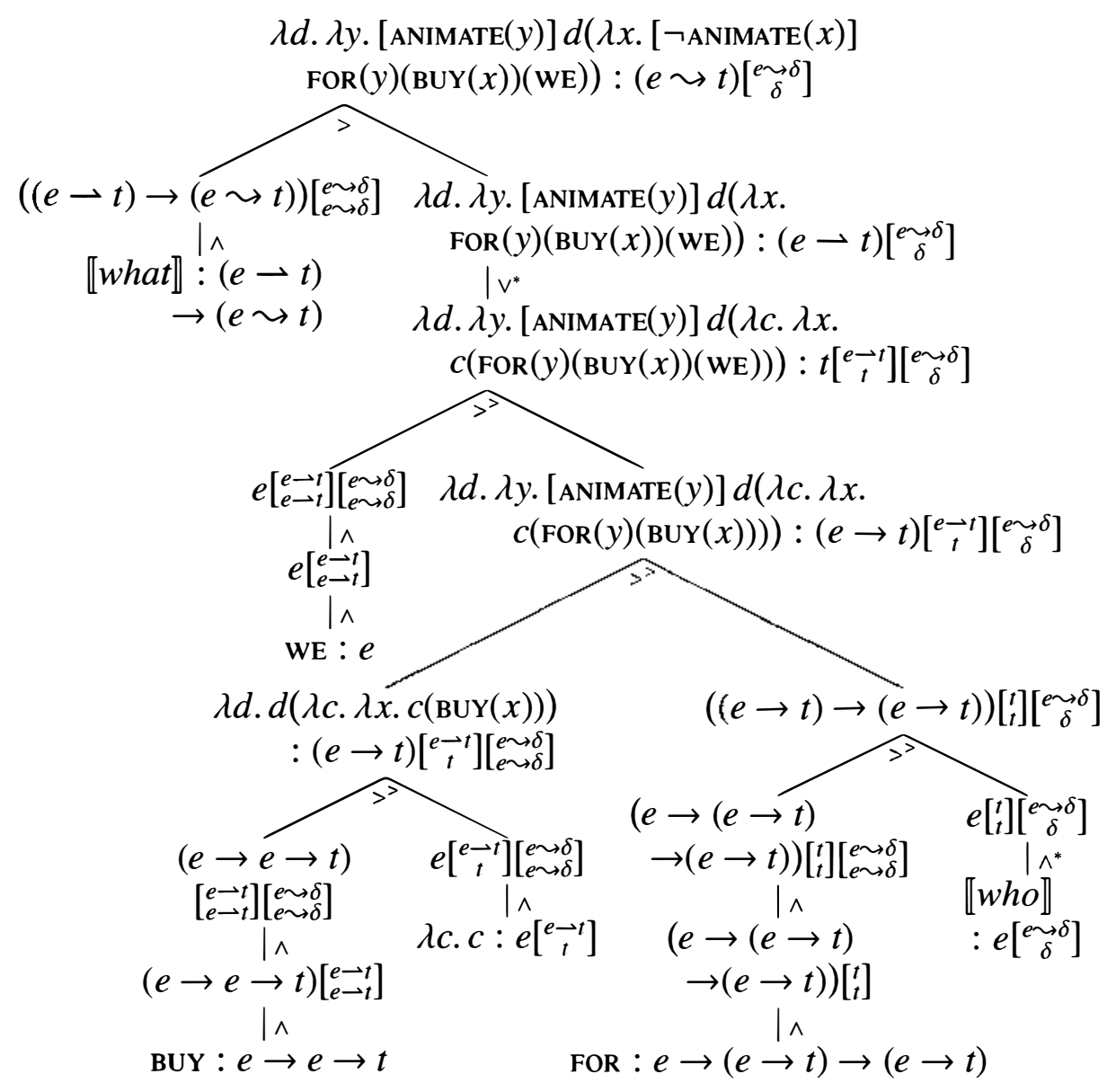

FIGURE 18. What we bought _for whom, with the wide-scope reading for whom

\subsection{Wide Scope and Baker's Ambiguity}

With second-order continuations, we can compute the wide-scope reading ( $3 a$ ) for the sentence $(1 \mathrm{~b})$ from Section 1, repeated here with gaps represented explicitly.

$\left(1 b^{\prime}\right)$ Who do you think _ remembers [what we bought _ for whom]?

I extend the grammar only by lifting it again as discussed above, and add no new denotations to the lexiçon other than the obvious missing entries

$$
\text { YOU }: e, \quad \text { THINK }: t \rightarrow e \rightarrow t \text {. }
$$

The wide-scope reading of the example (1b) is a double-wh question. Thus, we expect the matrix clause to have the semantic type $e \leadsto e \leadsto t$. What about the embedded clause? It acts locally like a single-wh question (what), but in addition should prepend " $e \sim$ " to the answer type (whom). We thus expect the embedded clause to have the type $(e \sim t)\left[\begin{array}{c}e \sim \delta \\ \delta\end{array}\right]$, a special case of which is $(e \sim t)\left[\begin{array}{c}e \leadsto t \\ t\end{array}\right]$.

Figure 18 shows a derivation for what we bought _for whom that culminates in precisely the expected type. The interesting part of the derivation is how the three 
continuation-manipulating elements what, , and whom enter it. The narrow-scope elements what and _ need to manipulate the inner answer type while remaining oblivious to the second continuation level, so we lift them. The wide-scope element whom, on the other hand, needs to manipulate the outer answer type while leaving the first continuation level alone; to achieve this effect, we lift it "from the inside" using the lifted lifting rule (depicted as $\wedge^{*}$ ). Near the top of the derivation, we use the lifted lowering rule (depicted as $\mathrm{V}^{*}$ ) to lower the embedded clause's denotation "from the inside", that is, on the first rather than second continuation level.

Given a meaning for what we bought _for whom of the expected type above, the semantics of the matrix question follows easily from the techniques already demonstrated in previous sections. Second-order continuations are no longer involved. The embedded clause is just a constituent that contains an in-situ $w h$-phrase whom; like any other such constituent, it combines with the rest of the sentence, including the raised $w h$-phrase $w h o$, to give a final denotation of type $e \leadsto e \leadsto t$, typical of a double-wh interrogative. Figure 19 shows the derivation.

We have seen that higher-order continuations allow in-situ wh-phrases to take wide scope. I now explain why raised $w h$-phrases cannot take wide scope, no matter how many times we lift the grammar. What does it mean for a raised wh-phrase to take wide scope? Based on the analyses so far, I make the following definitional characterization: A raised $w h$-phrase takes narrow scope when it contributes its " $e \sim$ " to the clause's value type, and wide scope when it contributes its " $e \sim$ " to the clause's outgoing answer type (or rather, one of the clause's outgoing answer types, in the presence of higher-order continuations). More precisely, consider a clause with a raised $w h$-phrase in front and a corresponding gap inside.

$$
[a[b \mathrm{wh}][c \ldots[d \mathrm{t}] \ldots]]
$$

For the $w h$-phrase $b$ to take narrow scope is for the clause $a$ to have semantic type of the form $(e \sim \alpha)\left[\begin{array}{l}\gamma_{1}^{\prime} \\ \gamma_{1}\end{array}\right] \cdots\left[\begin{array}{l}\gamma_{n}^{\prime} \\ \gamma_{n}\end{array}\right]$, and the clause-sans-wh-phrase $c$ the corresponding form $(e-\alpha)\left[\begin{array}{l}\gamma_{1}^{\prime} \\ \gamma_{1}\end{array}\right] \cdots\left[\begin{array}{l}\gamma_{n}^{\prime} \\ \gamma_{n}\end{array}\right]$, such that the " $e \sim$ " was contributed as " $e \rightarrow$ " by the gap $d$ and subsequently converted to " $e \sim$ " by the $w h$-phrase $b$. This is demonstrated in Section 3.2.

By contrast, for the $w h$-phrase $b$ to take wide scope is for $a$ to have semantic type of the form $\beta\left[\begin{array}{c}e \sim \alpha \\ \alpha\end{array}\right]\left[\begin{array}{c}\gamma_{1}^{\prime} \\ \gamma_{1}\end{array}\right] \cdots\left[\begin{array}{l}\gamma_{n}^{\prime} \\ \gamma_{n}\end{array}\right]$, and $c$ the corresponding form $\beta\left[\begin{array}{c}e-\alpha \\ \alpha\end{array}\right]\left[\begin{array}{c}\gamma_{1}^{\prime} \\ \gamma_{1}\end{array}\right] \cdots\left[\begin{array}{c}\gamma_{n}^{\prime} \\ \gamma_{n}\end{array}\right]$, such that the " $e \sim$ " was contributed as " $e-$ " by $d$ and subsequently converted to " $e \sim$ " by $b$. This is impossible because it requires replacing " $e \rightarrow$ " with " $e \sim$ " in an answer type, a feat performed by neither any element in the lexicon nor any rule in the grammar: A survey of the lexical items and grammar rules in this paper reveals that the they and their descendants-by-lifting only manipulate answer types by adding to them. Nothing ever takes apart any answer type until the answer has been lowered to value level. The formalism I use here does not stipulate thisin fact, one can easily introduce into the lexicon raised $w h$-phrases that take wide scope, but English does not appear to contain these denotations:

$$
\begin{gathered}
\llbracket w h a t \rrbracket \neq \lambda p \cdot \lambda c \cdot \lambda x .[\neg \operatorname{ANimate}(x)] p(c)(x): \alpha\left[\begin{array}{c}
e \rightarrow \gamma \\
\gamma
\end{array}\right] \rightarrow \alpha\left[\begin{array}{c}
e \sim \gamma \\
\gamma
\end{array}\right] \\
\llbracket w h o \rrbracket \neq \lambda p \cdot \lambda c \cdot \lambda x .[\operatorname{anim} \operatorname{ate}(x)] p(c)(x): \alpha\left[\begin{array}{c}
e \gamma \\
\gamma
\end{array}\right] \rightarrow \alpha\left[\begin{array}{c}
e \sim \gamma \\
\gamma
\end{array}\right]
\end{gathered}
$$




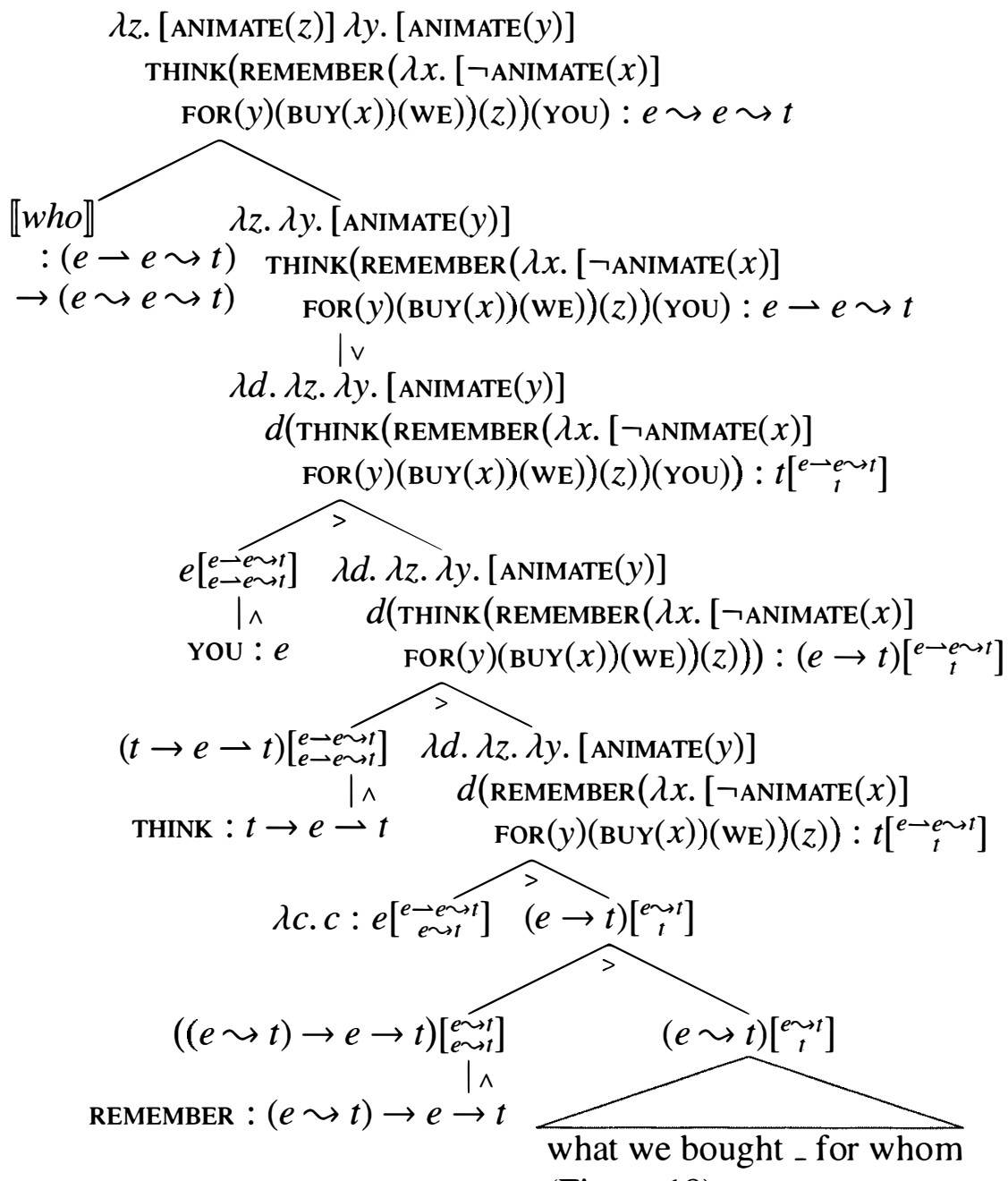

(Figure 18)

FIGURE 19. Who you thinks _ remembers what we bought _for whom, with the widescope reading for whom

\section{Discussion}

This paper presents a grammar fragment that captures two properties of English interrogatives: First, wh-phrases can appear both raised and in-situ. Second, insitu $w h$-phrases can take scope beyond the immediately enclosing clause, but raised wh-phrases must take scope exactly where they are pronounced. My fragment is inspired by Barker's continuation semantics for natural language (2000a; 2000b) and work on continuations and typed contexts in programming languages (Danvy and Filinski 1989, 1990; Murthy 1992; Wadler 1994).

As a semantics of interrogatives, this paper leaves many concerns unaddressed: languages other than English; pair-list questions; relative clauses; interactions with intensionality and quantification; and so on. Regardless, the two properties of interrogatives that I do explore fall out surprisingly naturally: The same $w h$-denotation works both raised and in-situ, but in raised position it is forced to take 
overt scope by a theorem of the type system. The basic ideas probably carry over to other kinds of so-called $\overline{\mathrm{A}}$-movement, such as topicalization. Continuations also suggest new ways to understand phenomena such as superiority and pied-piping, but for lack of space I leave these investigations for elsewhere.

\section{Endnotes}

*Thanks to Stuart Shieber, Chris Barker, Danny Fox, Pauline Jacobson, Norman Ramsey, Dylan Thurston, MIT 24.979 Spring 2001 (Kai von Fintel and Irene Heim), the Harvard AI Research Group, the Center for the Study of Language and Information at Stanford University, and the referees at SALT 12. This work is supported by National Science Foundation Grant IRI-9712068.

${ }^{1}$ I disregard here the distinction between questions that allow or expect PAIR-LIST answers (Alice remembers where we bought the vase, and Bob remembers where we bought the table) and questions that require or expect non-pair-list answers.

${ }^{2}$ To be clear, a raised $w h$-phrase can often take scope beyond the clause immediately enclosing its corresponding gap. The generalization I state here is that a raised $w h$-phrase cannot take scope beyond the clause in front of which it is pronounced.

${ }^{3}$ Throughout this paper, I use the Greek letters $\alpha, \beta, \gamma$, and $\delta$ to represent type variables, in other words variables that can be instantiated with any type. Thus what this paper calls types are known as TYPE-SCHEMES in the Hindley-Milner type system (Hindley and Seldin 1986). Also, by convention, all binary type constructors associate to the right: The type $e \rightarrow e \rightarrow t$, unparenthesized, means $e \rightarrow(e \rightarrow t)$.

${ }^{4}$ The concept of answer types in continuation semantics is separate from the concept of appropriate answerhood in interrogatives.

${ }^{5}$ So do Hendriks's (1993) and Barker's (2000a) later proposals. For simplicity and because they are irrelevant, I omit restrictions on quantification (“ANIMATE $(x) \Rightarrow \ldots$ ") in (10) and below.

${ }^{6}$ That I posit a phonologically null element is a matter of presentation and not critical to the approach to extraction sketched here. It would work equally well for my purposes to introduce type-shift operations that effectively roll [-】 into binary rules. ${ }^{7}$ The idea that a variable or gap is in some sense an identity function over continuations appeared in the work of Danvy and Filinski (1989, §3.4), and also has been mentioned to me by Barker. The more general idea that a variable or gap is an identity function of some sort has an even longer history in computer science (Hindley and Seldin 1986) and linguistics (Jacobson 1999).

${ }^{8}$ This pseudo-operational description is merely an intuitive sketch. The denotations in my semantics are computed purely in-situ according to local composition rules. ${ }^{9}$ In the same spirit, Barker (2000b) used higher-order continuations to treat widescope specific indefinites and interactions between coordination and antecedentcontained deletion. 


\section{References}

Baker, Carl Leroy. 1968. Indirect questions in English. Ph.D. thesis, University of Illinois.

Barker, Chris. 2000a. Continuations and the nature of quantification. Manuscript, University of California, San Diego; http://www. semanticsarchive. net/Archive/902ad5f7/.

2000b. Notes on higher-order continuations. Manuscript, University of California, San Diego.

Danvy, Olivier, and Andrzej Filinski. 1989. A functional abstraction of typed contexts. Tech. Rep. 89/12, DIKU, University of Copenhagen, Denmark. http://www.daimi .au.dk/ danvy/Papers/fatc.ps.gz. 1990. Abstracting control. In Proceedings of the 1990 ACM conference on Lisp and functional programming, 151-160. New York: ACM Press.

Epstein, Samuel David. 1992. Derivational constraints on $\bar{A}$-chain formation. Linguistic Inquiry 23(2):235-259.

Hendriks, Herman. 1993. Studied flexibility: Categories and types in syntax and semantics. Ph.D. thesis, Institute for Logic, Language and Computation, Universiteit van Amsterdam.

Hindley, J. Roger, and Jonathan P. Seldin. 1986. Introduction to combinators and $\lambda$-calculus. Cambridge: Cambridge University Press.

Jacobson, Pauline. 1999. Towards a variable-free semantics. Linguistics and Philosophy 22(2):117-184.

Kelsey, Richard, William Clinger, Jonathan Rees, et al. 1998. Revised ${ }^{5}$ report on the algorithmic language Scheme. Higher-Order and Symbolic Computation 11(1):7-105. Also as ACM SIGPLAN Notices 33(9):26-76.

Montague, Richard. 1974. The proper treatment of quantification in ordinary English. In Formal philoso phy: Selected papers of Richard Montague, ed. Richmond Thomason, 247-270. New Haven: Yale University Press.

Murthy, Chetan R. 1992. Control operators, hierarchies, and pseudo-classical type systems. In CW'92: Proceedings of the ACM SIGPLAN workshop on continuations, ed. Olivier Danvy and Carolyn Talcott, 49-71. Tech. Rep. STAN-CS-92-1426, Department of Computer Science, Stanford University. ftp://cstr.stanford.edu/pub/cstr/reports/cs/tr/92/1426.

Plotkin, Gordon D. 1975. Call-by-name, call-by-value and the $\lambda$-calculus. Theoretical Computer Science 1(2):125-159.

Steedman, Mark. 1987. Combinatory grammars and parasitic gaps. Natural Language and Linguistic Theory 5:403-439. 1996. Surface structure and interpretation. Cambridge: MIT Press.

Wadler, Philip. 1994. Monads and composable continuations. Lisp and Symbolic Computation 7(1):39-56. 